\title{
Experience Profiling of Fluorescence-Guided Surgery II: Non-Glioma Pathologies
}

\author{
So Young Ji', Jin Wook Kim", ${ }^{1,2}$ Chul-Kee Park ${ }^{1,2}$ \\ 'Department of Neurosurgery, Seoul National University Hospital, Seoul, Korea \\ 2Department of Neurosurgery, Seoul National University College of Medicine, Seoul, Korea
}

\author{
Received June 22, 2019 \\ Revised July 23, 2019 \\ Accepted July 30, 2019

\section{Correspondence} \\ Chul-Kee Park \\ Department of Neurosurgery, \\ Seoul National University Hospital, \\ Seoul National University \\ College of Medicine, \\ 101 Daehak-ro, Jongno-gu, \\ Seoul 03080, Korea \\ Tel: $+82-2-2072-0347$ \\ Fax: +82-504-154-4633 \\ E-mail: nschpark@snu.ac.kr
}

Background Only sporadic reports of fluorescence-guided surgery (FGS) have been published for non-glioma conditions. In this study, we focus on epidemiological data of fluorescence patterns and report the diverse experiences of FGS in non-gliomas.

Methods During 8.5 years between July 2010 and January 2019, 900 FGS for brain tumor performed in Seoul National University Hospital. Among them, a total of 73 histologically proven nonglioma patients were analyzed. Indications for FGS have been the possibility of anaplastic tumor in intra-axial tumors in preoperative MRI and an attempt to reproduce known anecdotal experiences of 5-Aminolevulinic Acid (5-ALA) fluorescence.

Results In cases of brain tumors except for gliomas, the most frequent cases were brain metastasis $(23$ cases) followed by lymphomas $(9$ cases) and meningeal tumors ( 8 cases). And there were embryonal tumors (6 cases), hemangioblastomas (4 cases), and solitary fibrous tumor/hemangiopericytomas (3 cases). Most brain metastases, meningiomas, primary central nervous system lymphomas, and treatment effect cases showed positive fluorescence. Moreover, some non-tumorous conditions also showed positive fluorescence. However, hemangioblastoma and germ cell tumor did not observe any fluorescence at all.

Conclusion 5-ALA induced fluorescence is not limited to glioma but is also evident in non-glioma and non-neoplastic conditions. This 5-ALA-induced fluorescence may be used as an intraoperative tool for various brain conditions.

Key Words Fluorescence; Surgery; Meningioma; Metastasis.

\section{INTRODUCTION}

Fluorescence-guided surgery (FGS) provides neurosurgeons with real-time, intraoperative information to distinguish malignant from normal or non-malignant tissue. Since FGS has high sensitivity and specificity to identify malignant glioma tissue, FGS is widely accepted for high grade gliomas and low grade gliomas with anaplastic foci [1]. However, the scope of use of FGS is extended beyond glioma surgery [2]. In particular, the usefulness of FGS for brain metastases and meningioma has been reported in several case series $[3,4]$. In

This is an Open Access article distributed under the terms of the Creative Commons Attribution Non-Commercial License (https://creativecommons.org/licenses/by-nc/4.0) which permits unrestricted non-commercial use, distribution, and reproduction in any medium, provided the original work is properly cited.

Copyright $\odot 2019$ The Korean Brain Tumor Society, The Korean Society for NeuroOncology, and The Korean Society for Pediatric Neuro-Oncology addition, it has been reported that it showed positive fluorescence outside brain tumors such as brain abscesses, spinal cord tumors, lung cancer and bladder cancer [5-8]. Experiences of incidental cases of FGS for various pathologies are accumulating rapidly. This report integrates our diverse and incidental experience of FGS in non-glial tumors and other anecdotal brain pathologies of their fluorescence patterns.

\section{MATERIALS AND METHODS}

During 8.5 years between July 2010 and January 2019, 900 FGS for brain tumor performed successively in Seoul National University Hospital. Among them, the fluorescence pattern of 827 glioma is reported in the previous article of this issue (Experience profiling of fluorescence-guided surgery I: gliomas). The other 73 FGSs have been proven histo- 
logically to be non-glial pathology. Indications for FGS are the possibility of anaplastic foci of intra-axial brain tumors in preoperative MRI or verification of previously reported sporadic applications (ex. hemangioblastoma). Application for extra-axial tumors such meningioma is the result of occasional ambiguous feature of morphologies in preoperative MRI. The Institutional Review Board of Seoul National University Hospital approved this retrospective study (IRB No: 1905-093-1034).

FGS methods and devices and fluorescence pattern analysis were the same as previously described. Briefly, $20 \mathrm{mg} / \mathrm{kg}$ of 5-Aminolevulinic Acid (5-ALA, Gliolan ${ }^{\circledR}$; Medac, Wedel, Germany) mixed with $50 \mathrm{~mL}$ of water 3 to 4 hours before the induction of general anesthesia, and FGS were done with a Leica M720 OH5 microscope (Leica, Wetzlar, Germany) equipped with an FL400 Fluorescence module or a Zeiss Pentero equipped with a fluorescent $400 \mathrm{~nm}$ UV light and filters (Zeiss, Oberkochen, Germany). Fluorescence intensity was made by optical judgement during the surgery and the red color was classified into "strong" intensity, pink into "weak" intensity, and blue into "no" fluorescence.

\section{RESULTS}

Of the 900 FGS performed, 73 (8.1\%) were histologically proven to be non-glioma lesions (Fig. 1). The most frequent application was brain metastasis (23 cases) followed by lymphoma ( 9 cases), meningeal tumor (8 cases), treatment effect (7 cases), embryonal tumor (6 cases), hemangioblastoma (4 cases), and solitary fibrous tumor/hemangiopericytoma (SFT/HPC, 3 cases). Other miscellaneous pathologies in- cluded non-tumorous tumefactive conditions such as reactive gliosis, demyelinating disease, and inflammatory disease.

\section{Brain metastasis}

Among 23 FGS cases of brain metastases, 20 cases (86.9\%) showed positive fluorescence including strong $(\mathrm{n}=6,26.1 \%)$ and weak fluorescence ( $\mathrm{n}=14,60.9 \%$ ) (Fig. 2A). Complete excision was performed in most cases except two cases with or without fluorescence. The reasons for incomplete resection in 2 cases were the tumor attachment to critical vessels and the stony hard contexture of the tumor. The primary sites of the cancer were lung $(n=15)$, colon $(n=3)$, ovary $(\mathrm{n}=2)$, kidney $(\mathrm{n}=1)$, and breast $(\mathrm{n}=1)$.

There were rare cases of brain metastasis from malignant peripheral nerve sheath tumor (MPNST) that showed strong fluorescence (Fig. 2B). A 35-year-old man with a history of MPNST resection at the left rib and lung, presented with headache and motor aphasia. Magnetic resonance imaging showed well-enhanced mass with surrounding edema and intratumoral hemorrhage in the left temporal lobe. Under the FGS, parts of the tumor showed strong fluorescence. Histological diagnosis of the mass was confirmed to be metastasis of MPNST.

\section{Meningioma and SFT/HPC}

We experienced 8 meningiomas of various WHO grades under FGS. Of them, 5 were fluorescence positive and the other 3 showed no fluorescence. A grade III anaplastic meningioma showed strong red fluorescence. However, one of the two grade II atypical meningiomas showed strong fluo-

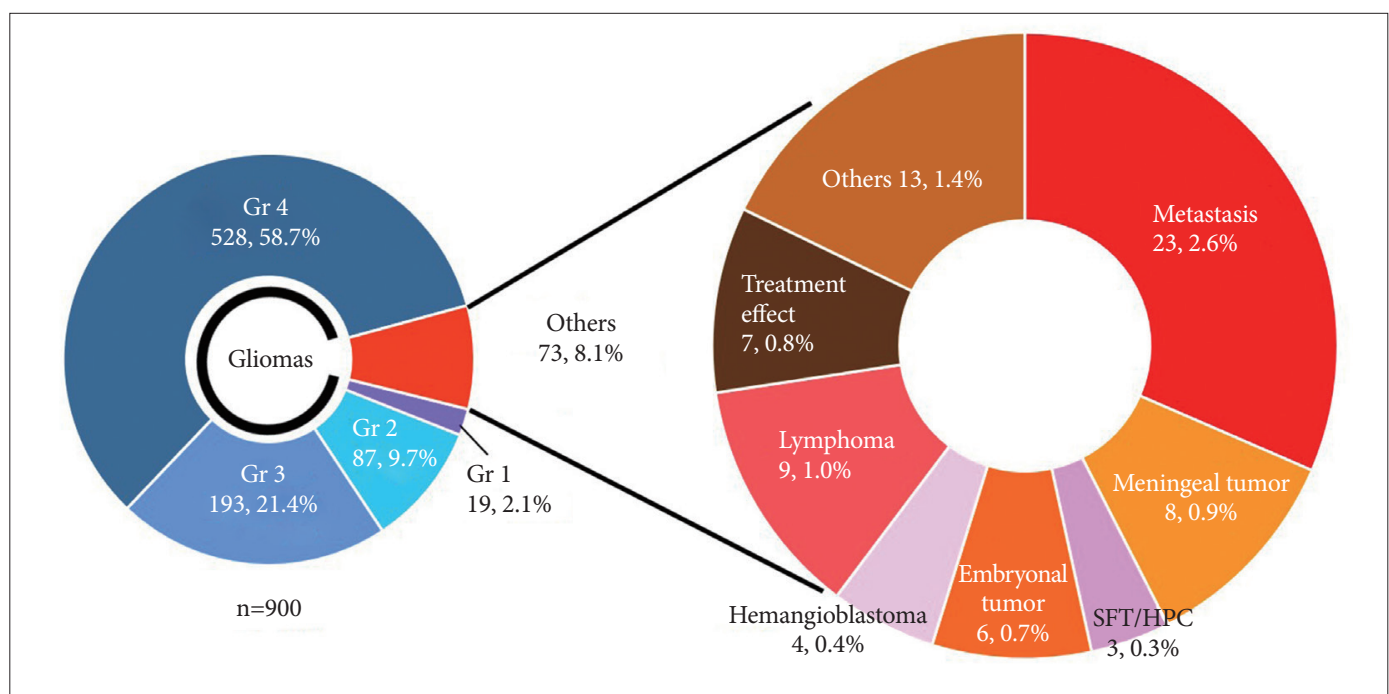

Fig. 1. Distribution of histological diagnosis of 73 fluorescence-guided surgery cases excluding glioma. Experience of 827 glioma cases is described in the other paper in this issue (Experience profiling of fluorescence-guided surgery I: gliomas). Numbers in the diagram indicate number of cases. SFT/HPC, solitary fibrous tumor/hemangiopericytoma; Gr, grade. 


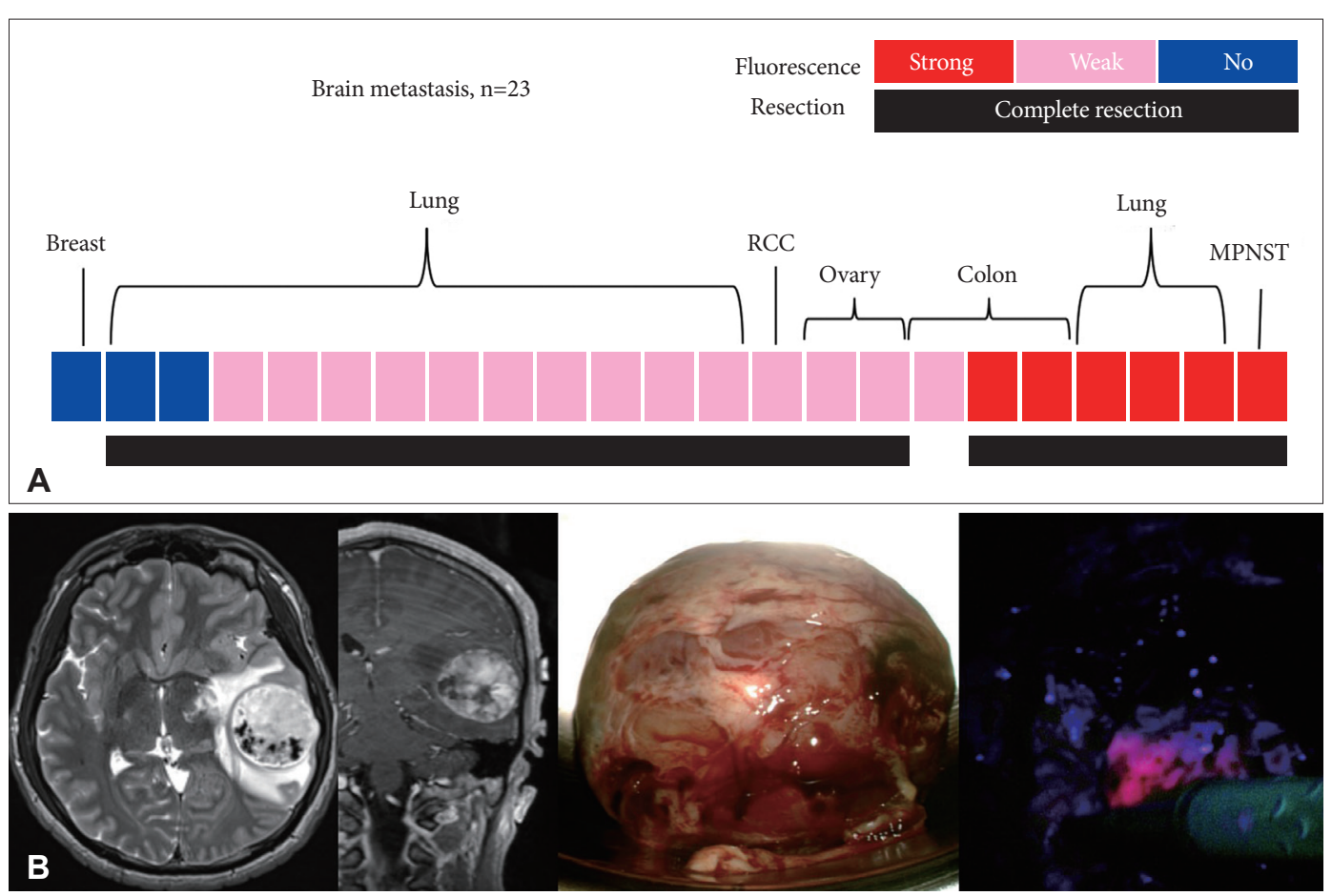

Fig. 2. A: Distribution of fluorescence intensity of 23 brain metastasis from various primary cancer sites. A single cubic unit denotes individual case. B: Illustrative case of brain metastasis of malignant peripheral nerve sheath tumor (MPNST). Transaxial T2-weighted and coronal T1-weighted MR images show a well enhancing round-shpaed mass with internal hemorrhage and peritumoral edema in left temporal lobe. The mass was excised in en bloc fashion and the part of the internal side of the mass showed strong red fluorescence. RCC, renal cell carcinoma.

rescence while the other showed no fluorescence. Among the remaining 5 grade I meningiomas, strong fluorescence was observed in 2 , weak fluorescence in 1 , and no fluorescence in 2. No histologic meningioma subtype predominance of fluorescence was observed.

All three SFT/HPC cases corresponding to WHO grade III were fluorescence positive. Strong positive was detected in 2 cases and weak fluorescence was observed in the other 1 case.

\section{Lymphoma and lymphoma-like lesions}

Sometimes it is difficult to make a differential radiological diagnosis between high-grade glioma and primary central nervous system lymphoma (PCNSL), even with FGS. Of 9 cases of PCNSL, 5 cases showed strong fluorescence as seen in glioblastoma and 2 cases showed weak fluorescence. The other 2 cases revealed no fluorescence.

Distinction between PCNSL and primary central nervous system angitiis (PCNSA) is even more challenging in the field of radiology and pathology. Unfortunately, FGS also does not do much for the differential diagnosis of those conditions. All 3 cases of PCNSA we experienced showed fluorescence positive (Fig. 3).

\section{Embryonal tumors and hemangioblastoma}

We have experienced 6 cases of embryonal tumors includ- ing germinoma ( 2 cases), medulloblastoma ( 2 cases), and pineoblastoma (2 cases). Only pineoblastomas showed weak fluorescence, whereas germinomas and medulloblastomas did not show any fluorescence. No fluorescence was observed in all 4 cases of hemangioblastomas as well.

\section{Treatment effect}

Treatment effect-related tisse fluorescence were confirmed in 7 cases. All the patients received FGS under the impression of recurrent glioblastoma. Although all cases showed positive fluorescence (Fig. 4), histologic examination revealed no viable tumor cells, mainly with radiation necrosis and reactive gliosis. The interval between radiation therapy and surgery varied from 1 month to 20 months.

\section{Non-tumorous conditions}

As FGS was applied for possible preoperative radiologic impression of high grade glioma, we accidentally experienced positive fluorescence for various non-neoplastic conditions.

A rare case of intracranial heterotopic ossification showed fluorescence positivity (Fig. 5A). A 51-year-old woman was hospitalized for dense calcification of the left frontal lobe with patch enhancement and peritumoral edema. Initial impression was oligodendrolioma with possible malignant 


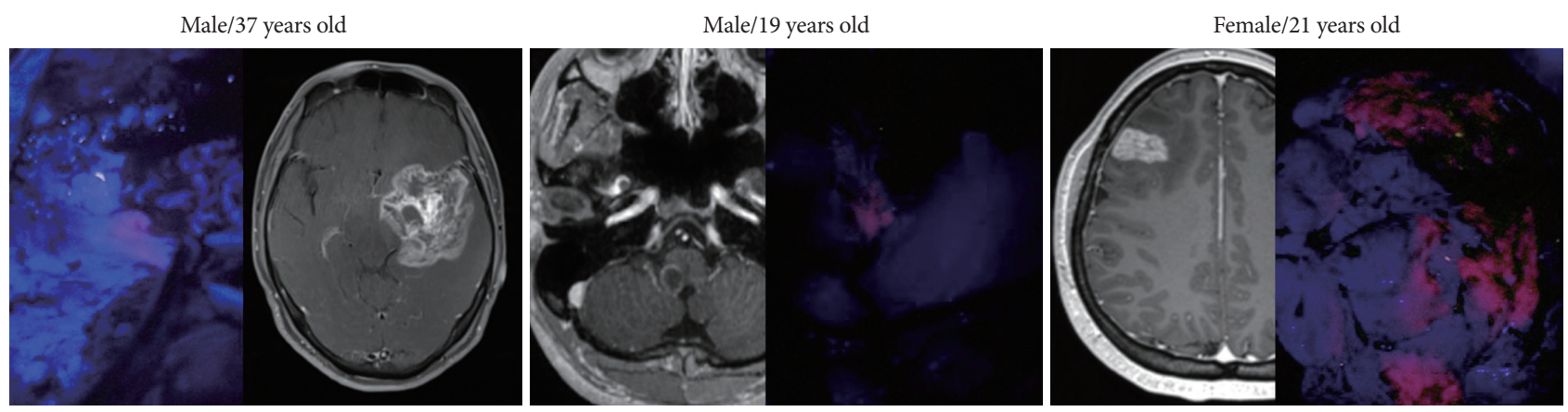

Fig. 3. Intraoperative photograph showing positive fluorescence and magnetic resonance images of 3 primary central nervous system angiitis cases.
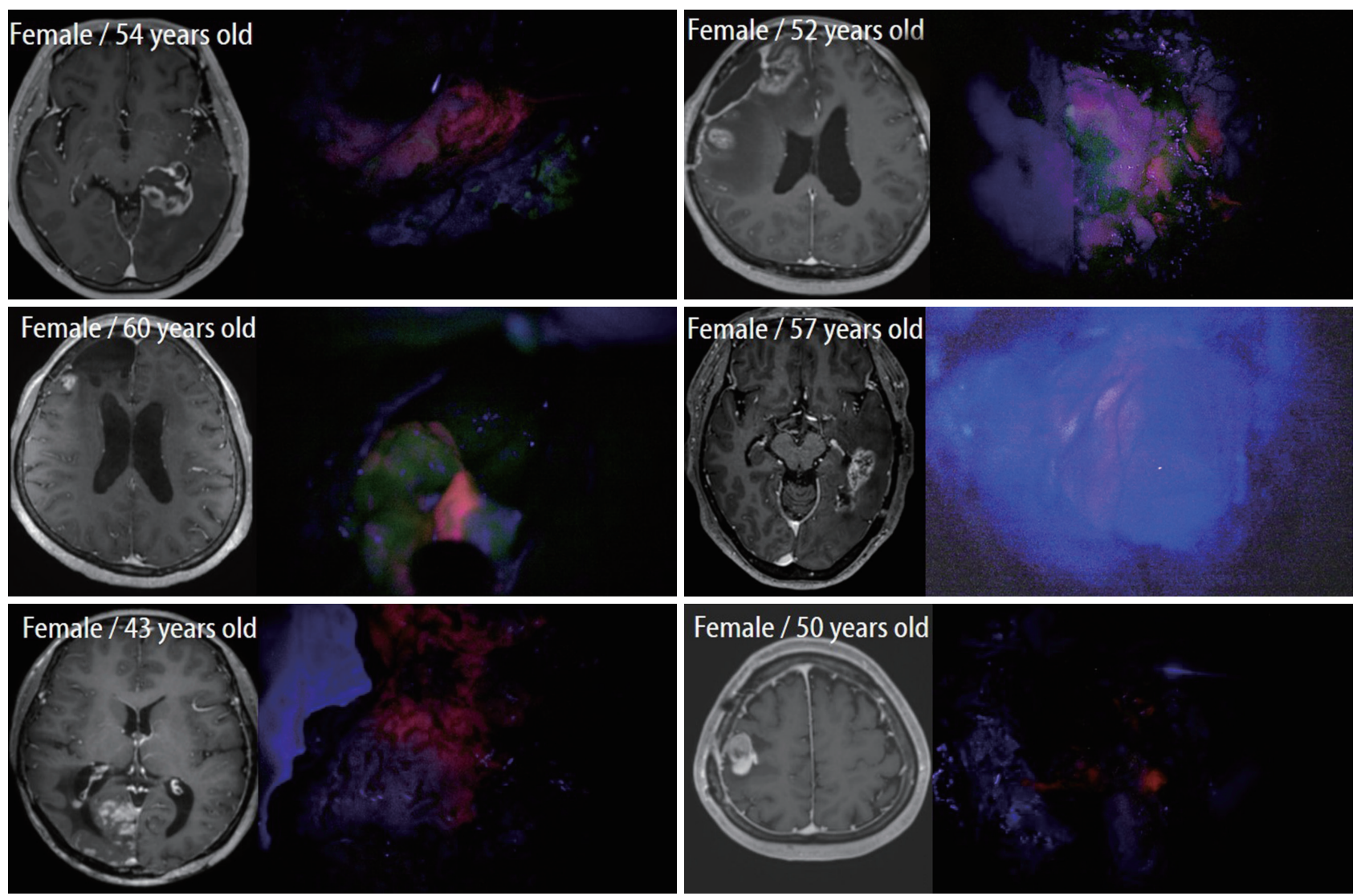

Fig. 4. Magenetic resonance images and intraoperative fluorescence pattern of treatment effect after radiotherapy. Positive fluorescence areas were observed although no viable tumor cells were idendtified in histological examination.

transformation. During the FGS, strong fluorescence was observed. However, histological investigation showed reactive gliosis with numerous Rosenthal fibers and diffuse calcification. The final histological diagnosis was heterotopic ossification without any evidence of neoplasm.

A case of glioependymal cyst showed strong positivity of fluorescence (Fig. 5B). This 69-year old female had a small enhancing cystic lesion at the left peri-trigonal which have grown slowly for 3 years. Under the impression of tumorous condition, FGS was performed and strong fluorescence was identified in the solid part of the mass. However, the lesion was diagnosed as a glioependymal cyst without any neoplastic evidence. Histopathological analysis showed ciliated ependymal linings with underlying reactive gliosis and perivascular lymphoplasmacytic infiltration.

Inflammatory conditions could exhibit positive fluorescence. Histologically confirmed chronic active inflammation and meningoencephalitis mimicking tumorous condition in radiological studies showed strong red fluorescence around the lesion of interest (Fig. 5C). However, we also did not experience any discernable fluorescence in conditions with histologically confirmed inflammatory pseudotumors, reactive 


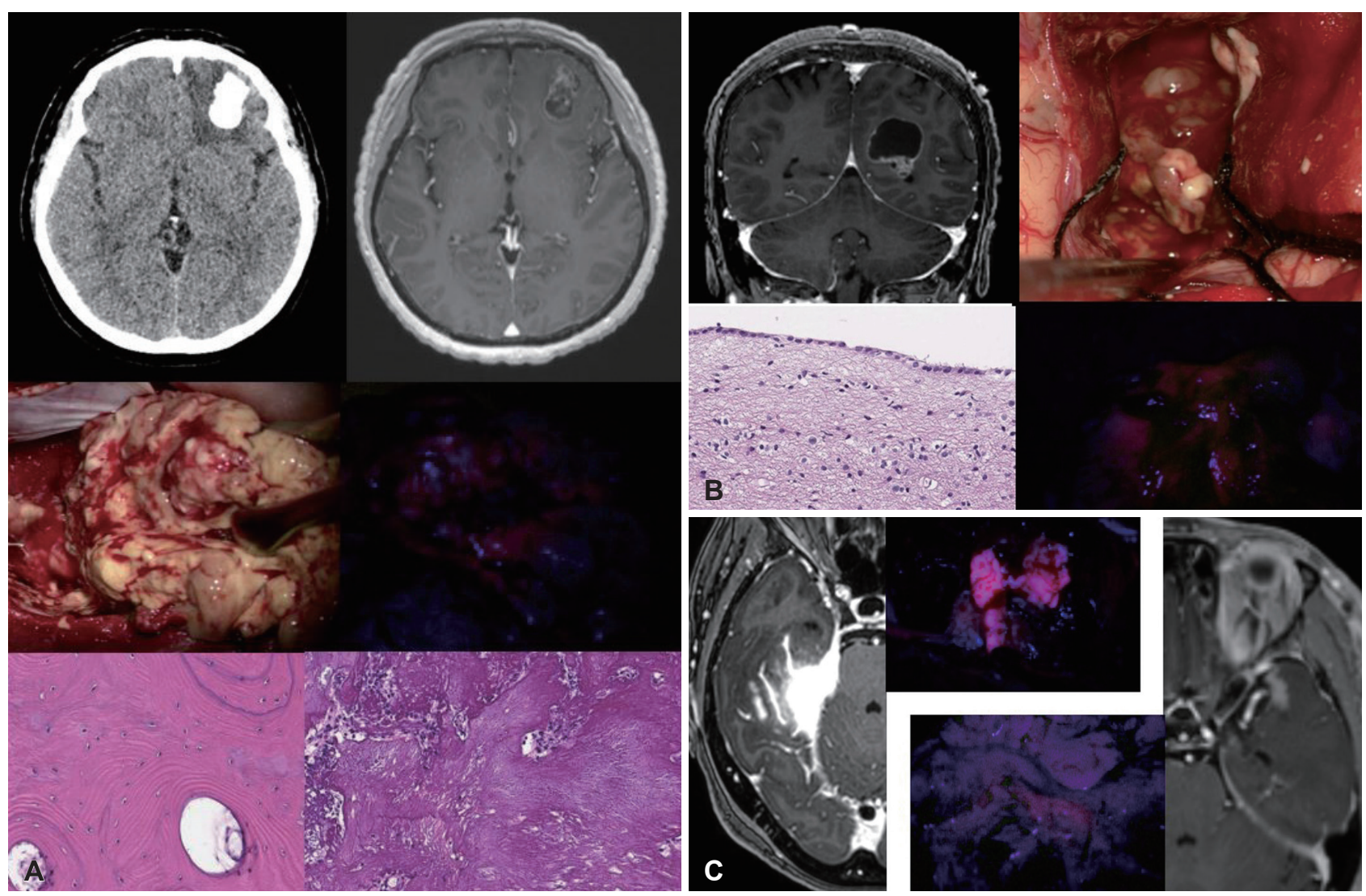

Fig. 5. Examples of non-tumorous conditions showing positive fluorescenase. A: A case of intracranial heterotopic ossification. Non-contrast computed tomography shows dense calcific lesion in the left frontal lobe.The lesion is irregulary enhanced in MRI. Intraoperative finding shows strong fluorescence. Histologically, the mass is composed of calcification with numerous Rosenthal fibers. B: A case of glioependymal cyst. Preoperative MRI shows an enhanced mass lesion with cyst in the left periventricular white matter. The solid portion of resected mass exhibit strong fluorescence. Histological finding denies any evidence of neoplasm but for only showing reactive gliosis with ciliated ependymal linings. A, B: Hematoxylin and eosin stain; original magnification $\times 400$. C: Cases of pachymeningitis (left) and meningoencephlitis (right) which shows positive fluorescence.

gliosis for various reasons, and demyelinating diseases.

\section{DISCUSSION}

Retrospective studies have reported that metastatic brain tumors have a broad range of fluorescence signals from $30 \%$ to $80 \%$ [9-11]. In our data, the incidence of fluorescence positive for brain metastases was even higher, reaching $86.9 \%$. However, there is no specific relationship between the primary cancer type and the fluorescence intensity $[11,12]$. In brain metastases, it is reported that the fluorescence in brain metastases is unevenly distributed at the same histology and origin, as expression of ferrochilatase, which plays an important role in PpIX accumulation in glioblastoma, varies inbrain metastasis $[11,13,14]$. On the other hand, we experienced the same experience as previous reports mentioning that diffuse fluorescence may be observed in the peri-edema area of brain metastases irrespective of the presence of tumor cells $[10,15]$. Thus, unlike malignant gliomas, weakly positive fluorescent areas in the peritumoral area should be carefully removed to account for false positive results in brain metastases. For this reason, it has been reported that fluorescence of brain metastasis by 5 -ALA is not significantly associated with the degree of surgical resection [9]. Nevertheless, FGS for brain metastases enables improved surgical outcome with respect to tumor local control rate and progression free survival $[11,13]$.

Visible fluorescence has been reported in $77-96 \%$ of meningiomas in most studies $[12,16-22]$. The fluorescence patterns of meningiomes have proven to be uniform and strong in most cases $[4,16,23]$. Interestingly, as can be seen from our experience, the fluorescence intensity of meningiomes is not highly correlated with histologic features or malignant tumors [22,23]. The specific mechanism of 5-ALA fluorescence in meningioma have never been studied. However, the role of the disrupted brain blood barrier is not a major determinant of 5-ALA uptake in meningioma [3,20]. Recent case reports suggest that FGS in meningioma is a useful and promising tool for intraoperative visualization of meningioma tissue and detection of bone infiltration $[22,24]$. Some expe- 
riences in meningiomas also suggest that FGS can distinguish between hypertrophic dura and tumor-infiltrated dura $[19,22,25]$. FGS can make it possible to reduce the recurrence rate of meningiomas by identifying residual tumors in the adjacent dura, bone flaps and brain parenchyma.

Although 5-ALA-induced fluorescence has been demonstrated in most lymphomas ( $75 \%$ to $100 \%$ ), the exact mechanism of 5-ALA metabolism and accumulation of fluorescent PpIX in PCNSL cells remains unknown [26,27]. Several studies have suggested that a proton-coupled folate transporter, one of the membrane transporters of lymphoma cells, is associated with the uptake of 5-ALA in PCNSL, and the lack of ferrochelatase enzyme activity in malignant lymphoma is associated with the accumulation of fluorescent PpIX [28-32]. 5-ALA-induced fluorescence in PCNSL is mostly observed with strong intensity and uniform pattern. In PCNSL, histologic confirmation is essential for proper diagnosis and treatment, such as chemotherapy and radiation therapy. According to the histopathological analysis of the literature, the fluorescent tissue in PCNSL contains well diagnostic tissue with high positive predictive value $[27,33]$. Thus, 5-ALA induced fluorescence can be a potentially useful intraoperative tool for identifying diagnostic lymphoma tissue in biopsies of PCNSL.

Hemangioblastoma has been reported to show positive fluorescence in mural nodule $[34,35]$. However, we could not detect any fluorescence in hemangioblastoma under the general setting of FGS condition. Furthermore, although there is a literature reporting positive fluorescence, we were unable to detect any fluorescence in germ cell tumors [36].

After adjuvant therapy for the treatment of malignant glioma, brain tissue expereiences reactive and inflammatory changes such as gliosis, mesenchymal scar formation, and vascular hyalinization [37-41]. These changes can cause degenerative tissues to become fluorescent positive after adjuvant therapy. In particular, radiation disrupts the blood-brain barrier and induces inflammatory cell infiltration associated with 5-ALA uptake and accumulation of fluorescent PpIX. Since the effects of radiation change with time, some literature suggests that fluorescence may be related to the interval between radiation therapy and surgery mentioning weak recurrent lesions become apparent within 4 to 9 months after radiation without tumor recurrence $[37,42,43]$. However, in our data, fluorescence intensity is not significantly associated with the interval between radiation therapy and surgery $[37,44]$. It should be kept in mind that intraoperative fluorescence alone is not enough to distinguish between treatment effects and tumor recurrence.

5-ALA-induced fluorescence is not limited to malignancy. To date, 5-ALA-induced fluorescence has been reported in several cases of brain abscess, multiple sclerosis, demyelinat- ing disease, radiation necrosis, and cerebral infarction [4547]. In the non-tumorous condition, the expression patterns of key enzymes and transporters involved in the fluorescent PpIX biosynthetic pathway are different from the tumor. According to the literature, 5-ALA-induced fluorescence is presumed to be associated with reactive astrocytes and inflammation [46,48]. Inflammation causes a reaction of immune cell infiltration involving macrophages, lymphocytes, and neutrophils. Some studies have reported that macrophages and activated lymphocytes have high expression of peptide transporter 1 (PEPT1) and low expression of ferrochelatase (FECH) in PpIX biosynthesis pathway, leading to the accumulation of fluorescent PpIX [32,48]. Thereby, intraoperative fluorescence is observed in inflammation or infection.

In conclusion, 5-ALA-induced fluorescence is not limited to glioma but is also evident in non-glioma and non-neoplastic conditions. Further studies should establish the utility of FGS in various areas, as the biosynthetic mechanism of fluorescent PpIX in non-glioma tumors has not yet been elucidated.

\section{Conflicts of Interest}

The authors have no potential conflicts of interest.

\section{Acknowledgments}

This research was supported by the Bio \& Medical Technology Development Program of the National Research Foundation (NRF) funded by the Ministry of Science \& ICT (NRF-2018M3A9H3021707) in Korea.

\section{REFERENCES}

1. Hadjipanayis CG, Widhalm G, Stummer W. What is the surgical benefit of utilizing 5 -aminolevulinic acid for fluorescence-guided surgery of malignant gliomas? Neurosurgery 2015;77:663-73.

2. Ewelt C, Nemes A, Senner V, et al. Fluorescence in neurosurgery: Its diagnostic and therapeutic use. Review of the literature. J Photochem Photobiol B 2015;148:302-9.

3. Chae MP, Song SW, Park SH, Park CK. Experience with 5-aminolevulinic acid in fluorescence-guided resection of a deep sylvian meningioma. J Korean Neurosurg Soc 2012;52:558-60.

4. Valdes PA, Bekelis K, Harris BT, et al. 5-Aminolevulinic acid-induced protoporphyrin IX fluorescence in meningioma: qualitative and quantitative measurements in vivo. Neurosurgery 2014;10 Suppl 1:74-82; discussion 82-3.

5. Grimbergen MC, van Swol CF, van Moorselaar RJ, Uff J, MahadevanJansen A, Stone N. Raman spectroscopy of bladder tissue in the presence of 5-aminolevulinic acid. J Photochem Photobiol B 2009;95:170-6.

6. Huber RM, Gamarra F, Hautmann H, et al. 5-aminolaevulinic acid (ALA) for the fluorescence detection of bronchial tumors. Diagn Ther Endosc 1999;5:113-8.

7. Baas P, Triesscheijn M, Burgers S, van Pel R, Stewart F, Aalders M. Fluorescence detection of pleural malignancies using 5 -aminolaevulinic acid. Chest 2006;129:718-24.

8. Millesi M, Kiesel B, Woehrer A, et al. Analysis of 5-aminolevulinic acid-induced fluorescence in 55 different spinal tumors. Neurosurg Focus 2014;36:E11.

9. Kamp MA, Munoz-Bendix C, Mijderwijk HJ, et al. Is 5-ALA fluorescence of cerebral metastases a prognostic factor for local recurrence and overall survival? J Neurooncol 2019;141:547-53. 
10. Utsuki S, Miyoshi N, Oka H, et al. Fluorescence-guided resection of metastatic brain tumors using a 5 -aminolevulinic acid-induced protoporphyrin IX: pathological study. Brain Tumor Pathol 2007;24:53-5.

11. Kamp MA, Grosser P, Felsberg J, et al. 5-aminolevulinic acid (5-ALA)induced fluorescence in intracerebral metastases: a retrospective study. Acta Neurochir (Wien) 2012;154:223-8; discussion 228.

12. Marbacher S, Klinger E, Schwyzer L, et al. Use of fluorescence to guide resection or biopsy of primary brain tumors and brain metastases. Neurosurg Focus 2014;36:E10.

13. Kamp MA, Fischer I, Bühner J, et al. 5-ALA fluorescence of cerebral metastases and its impact for the local-in-brain progression. Oncotarget 2016;7:66776-89.

14. Teng L, Nakada M, Zhao SG, et al. Silencing of ferrochelatase enhances 5 -aminolevulinic acid-based fluorescence and photodynamic therapy efficacy. Br J Cancer 2011;104:798-807.

15. Utsuki S, Oka H, Sato S, et al. Histological examination of false positive tissue resection using 5-aminolevulinic acid-induced fluorescence guidance. Neurol Med Chir (Tokyo) 2007;47:210-3; discussion 213-4.

16. Valdes PA, Millesi M, Widhalm G, Roberts DW. 5-aminolevulinic acid induced protoporphyrin IX (ALA-PPIX) fluorescence guidance in meningioma surgery. J Neurooncol 2019;141:555-65.

17. Potapov AA, Goryaynov SA, Okhlopkov VA, et al. Laser biospectroscopy and 5-ALA fluorescence navigation as a helpful tool in the meningioma resection. Neurosurg Rev 2016;39:437-47.

18. Morofuji Y, Matsuo T, Hayashi Y, Suyama K, Nagata I. Usefulness of intraoperative photodynamic diagnosis using 5 -aminolevulinic acid for meningiomas with cranial invasion: technical case report. Neurosurgery 2008;62 (3 Suppl 1):102-3; discussion 103-4.

19. Kajimoto Y, Kuroiwa T, Miyatake S, et al. Use of 5-aminolevulinic acid in fluorescence-guided resection of meningioma with high risk of recurrence. Case report. J Neurosurg 2007;106:1070-4.

20. Coluccia D, Fandino J, Fujioka M, Cordovi S, Muroi C, Landolt H. Intraoperative 5-aminolevulinic-acid-induced fluorescence in meningiomas. Acta Neurochir (Wien) 2010;152:1711-9.

21. Cornelius JF, Slotty PJ, Kamp MA, Schneiderhan TM, Steiger HJ, ElKhatib M. Impact of 5-aminolevulinic acid fluorescence-guided surgery on the extent of resection of meningiomas--with special regard to high-grade tumors. Photodiagnosis Photodyn Ther 2014;11:481-90.

22. Foster N, Eljamel S. ALA-induced fluorescence image guided surgery of meningiomas: a meta-analyses. Photodiagnosis Photodyn Ther 2016;15:73-8

23. Millesi M, Kiesel B, Mischkulnig M, et al. Analysis of the surgical benefits of 5-ALA-induced fluorescence in intracranial meningiomas: experience in 204 meningiomas. J Neurosurg 2016;125:1408-19.

24. Della Puppa A, Rustemi O, Gioffrè G, et al. Predictive value of intraoperative 5-aminolevulinic acid-induced fluorescence for detecting bone invasion in meningioma surgery. J Neurosurg 2014;120:840-5.

25. Valdés PA, Leblond F, Kim A, et al. Quantitative fluorescence in intracranial tumor: implications for ALA-induced PpIX as an intraoperative biomarker. J Neurosurg 2011;115:11-7.

26. Yamamoto J, Kitagawa T, Akiba D, Nishizawa S. 5-Aminolevulinic acid-induced fluorescence in cerebellar primary central nervous system lymphoma: a case report and literature review. Turk Neurosurg 2015;25:796-800.

27. Kiesel B, Millesi M, Woehrer A, et al. 5-ALA-induced fluorescence as a marker for diagnostic tissue in stereotactic biopsies of intracranial lymphomas: experience in 41 patients. Neurosurg Focus 2018;44:E7.

28. Takada T, Tamura M, Yamamoto T, Matsui H, Matsumura A. Selective accumulation of hematoporphyrin derivative in glioma through protoncoupled folate transporter SLC46A1. J Clin Biochem Nutr 2014;54:26-30.

29. Desmoulin SK, Hou Z, Gangjee A, Matherly LH. The human proton- coupled folate transporter: Biology and therapeutic applications to cancer. Cancer Biol Ther 2012;13:1355-73.

30. Evers G, Kamp M, Warneke N, et al. 5-Aminolaevulinic Acid-Induced Fluorescence in Primary Central Nervous System Lymphoma. World Neurosurg 2017;98:375-80.

31. El-Sharabasy MM, el-Waseef AM, Hafez MM, Salim SA. Porphyrin metabolism in some malignant diseases. Br J Cancer 1992;65:409-12.

32. Rittenhouse-Diakun K, Van Leengoed H, Morgan J, et al. The role of transferrin receptor (CD71) in photodynamic therapy of activated and malignant lymphocytes using the heme precursor delta-aminolevulinic acid (ALA). Photochem Photobiol 1995;61:523-8.

33. Yamamoto T, Ishikawa E, Miki S, et al. Photodynamic diagnosis using 5-aminolevulinic acid in 41 biopsies for primary central nervous system lymphoma. Photochem Photobiol 2015;91:1452-7.

34. Utsuki S, Oka H, Kijima C, Miyajima Y, Hagiwara H, Fujii K. Utility of intraoperative fluorescent diagnosis of residual hemangioblastoma using 5-aminolevulinic acid. Neurol India 2011;59:612-5.

35. Utsuki S, Oka H, Sato K, Shimizu S, Suzuki S, Fujii K. Fluorescence diagnosis of tumor cells in hemangioblastoma cysts with 5-aminolevulinic acid. J Neurosurg 2010;112:130-2.

36. Takeda J, Nonaka M, Li Y, et al. 5-ALA fluorescence-guided endoscopic surgery for mixed germ cell tumors. J Neurooncol 2017;134:119-24.

37. Kamp MA, Felsberg J, Sadat H, et al. 5-ALA-induced fluorescence behavior of reactive tissue changes following glioblastoma treatment with radiation and chemotherapy. Acta Neurochir (Wien) 2015;157:207-13; discussion 213-4.

38. Giglio P, Gilbert MR. Cerebral radiation necrosis. Neurologist 2003;9:180-8.

39. Chaskis C, Neyns B, Michotte A, De Ridder M, Everaert H. Pseudoprogression after radiotherapy with concurrent temozolomide for high-grade glioma: clinical observations and working recommendations. Surg Neurol 2009;72:423-8.

40. Brandes AA, Tosoni A, Spagnolli F, et al. Disease progression or pseudoprogression after concomitant radiochemotherapy treatment: pitfalls in neurooncology. Neuro Oncol 2008;10:361-7.

41. De Wit MC, de Bruin HG, Eijkenboom W, Sillevis Smitt PA, van den Bent MJ. Immediate post-radiotherapy changes in malignant glioma can mimic tumor progression. Neurology 2004;63:535-7.

42. Wong CS, Van der Kogel AJ. Mechanisms of radiation injury to the central nervous system: implications for neuroprotection. Mol Interv 2004;4:273-84.

43. Brandsma D, Stalpers L, Taal W, Sminia P, van den Bent MJ. Clinical features, mechanisms, and management of pseudoprogression in malignant gliomas. Lancet Oncol 2008;9:453-61.

44. Nabavi A, Thurm H, Zountsas B, et al. Five-aminolevulinic acid for fluorescence-guided resection of recurrent malignant gliomas: a phase ii study. Neurosurgery 2009;65:1070-6; discussion 1076-7.

45. Behling F, Hennersdorf F, Bornemann A, Tatagiba M, Skardelly M. 5-Aminolevulinic acid accumulation in a cerebral infarction mimicking high-grade glioma. World Neurosurg 2016;92:586.e5-e8.

46. Nestler U, Warter A, Cabre P, Manzo N. A case of late-onset multiple sclerosis mimicking glioblastoma and displaying intraoperative 5-aminolevulinic acid fluorescence. Acta Neurochir (Wien) 2012;154:899-901.

47. Höhne J, Brawanski A, Schebesch KM. Fluorescein sodium-guided surgery of a brain abscess: a case report. Surg Neurol Int 2016;7(Suppl 39):S955-7.

48. Omoto K, Matsuda R, Nakagawa I, Motoyama Y, Nakase H. Falsepositive inflammatory change mimicking glioblastoma multiforme under 5-aminolevulinic acid-guided surgery: a case report. Surg Neurol Int 2018;9:49. 